\title{
Extragastrointestinal Stromal Tumor - report of a rare case
}

\author{
Alexandra Mesquita ${ }^{1}$, Fátima Braga ${ }^{1}$, Angeles Peteiro ${ }^{2}$, Carlos Sottomayor ${ }^{1}$ \\ 'Department of Oncology Hospital Pedro Hispano Portugal \\ ${ }^{2}$ Anatomic Pathology Service Hospital Pedro Hispano Portugal
}

\begin{abstract}
Despite the name, gastrointestinal stromal tumor (GIST) is a mesenchymal gastrointestinal neoplasm that may occur outside the gastrointestinal tract. The correct characterization based on histological features and immunohistochemical expression of c-kit (CD117) has allowed targeted treatment for this kind of cancer and therefore improvements in the outcome.
\end{abstract}

The authors present a rare clinical case of a GIST of the pelvic cavity with a long 10 years follow-up.

Keywords:. Gastrointestinal stromal tumor. CD117. Imatinib

Palabras clave: Tumor estromal gastrointestinal. CD117. Imatinib.

\section{Introduction}

Gastrointestinal Stromal Tumors (GISTs) are the most common mesenchymal neoplasms of the gastrointestinal tract. The most common localization is gastric, however they may be present in extra-gastrointestinal locations. They arise from the Cajal cells, which are responsible for the gastrointestinal motility ${ }^{1}$. In 95\% of the cases there is c-KIT (CD117) activation in the tumor cells, and therefore the immunohistochemical and molecular characterization is essential to the correct diagnosis ${ }^{2}$. The identification of mutations mostly in exon 11 and or in exons 9 and 13 of the c-kit proto-oncogene coding for c-kit (CD117) in many GISTs, has resulted in a better understanding of their oncogenic mechanisms. The finding of remarkable antitumor effects of the molecular inhibitor Imatinib as adjuvant therapy and metastatic inoperable GISTs, has enabled a better survival ${ }^{3}$.

\section{Case Report}

In February 2003, a 53 year- old woman with no significant medical history presented at the Emergency Department with complaints of abdominal pain and vomiting. There was no history of fever, fatigue or weight loss. Physical examination with no tenderness and no palpable masses. No adenopathies were detected. Blood testing showed Hemoglobin (Hb) $10.4 \mathrm{~g} / \mathrm{dl}$, Mean Corpuscular Volume (MCV) $71 \mathrm{fL}$, Mean Corpuscular Hemoglobin (MCH) 21 pg, normal leucogram and platelets, no renal or liver disfunction, normal inflammatory markers. Abdominal CT scan showed a large pelvic mass. She underwent an exploratory laparotomy which confirmed the presence of an abdominal mass in contact with the anterior wall of the uterus, without invasion of the myometrium but invading the adnexal structures and the appendix (figure 1). A total hysterectomy and resection of vesical uterine peritoneum and appendicectomy was carried out.

Histological examination showed a diffusely growing tumor composed of polygonal cells with ample eosinophilic cytoplasm giving them an epithelioid appearance, many with intracytoplasmic inclusions. Mitotic activity was 5 mitoses per 50 high-power fields. The tumor cells showed diffuse and strong immunoreactivity for CD117 (figure 2) and CD34, and vimentin was expressed with accentuation of staining in the cytoplasmic inclusion. They were negative for CK,
EMA, SMA, S-100, Calretinin and HBME1. The ovaries were normal. Uterus and appendix showed no involvement other than that of the serosa. We concluded that the tumor arose in the pelvic peritoneum. At this time the tumor was diagnosed as sarcoma with rhabdoid phenotype. The patient started chemotherapy with Ifosfamide $250 \mathrm{mg}$, Mesna 600mg, Epirrubicin 90mg and dexamethasone 10mg.

The case was sent to the Prof. Christopher Fletcher for a second opinion at Brigham and Women's Hospital. The final diagnosis was GIST with a high risk of aggressive behavior.

While recovering from the first cycle of chemotherapy, the abdominal pain became more intense and she was re-admitted after an episode of syncope and marked hypotension.

Blood tests documented anemia ( $\mathrm{Hb} 7.1 \mathrm{~g} / \mathrm{dl}$, MCV $85.5 \mathrm{fL}, \mathrm{MCH}$ $32.4 \mathrm{pg})$. She underwent another emergent exploratory laparotomy which showed an extensive intra-peritoneal bleed. New peritoneal biopsies were performed and confirmed metastatic GIST and resection with microscopic residual disease (R1) was carried out. Genetic testing showed exon 11 mutation. PET scan showed positive mediastinal nodes. Palliative chemotherapy with Imatinib Mesylate 400mg/day was started in January 2004, assuming residual disease. The patient maintains therapy with Imatinib $400 \mathrm{mg} /$ day for approximately 10 years without any side effects. The last CT scan in October 2015, showed no evidence of recurrence of the disease and the blood tests remain normal.

Figure1. CT scan os abdominal cavity

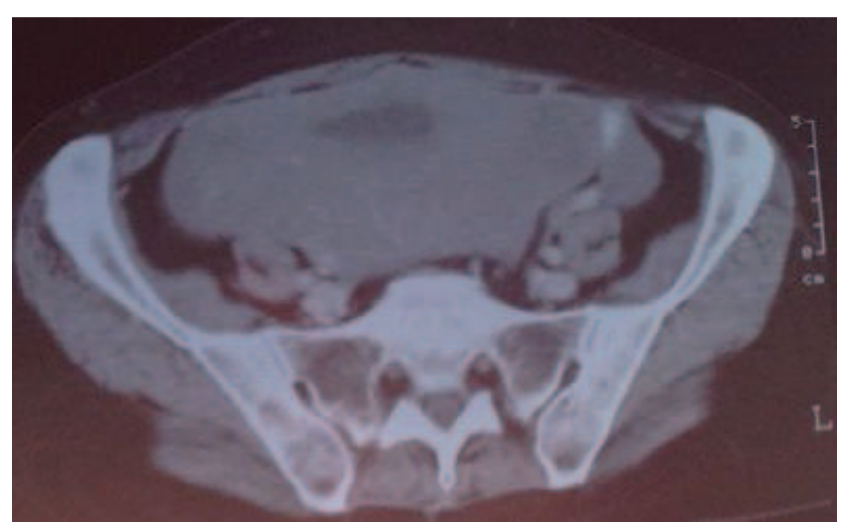


Figure 2. EGIST - CD117 positive

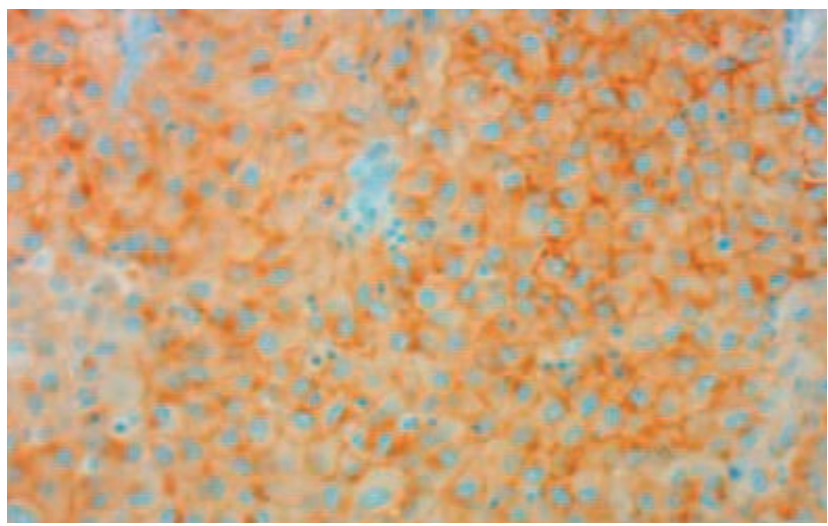

\section{Discussion}

Gastrointestinal stromal tumor (GIST) is identified in the fifth or sixth decades of life ${ }^{1}$. It may be detected at any site along the gastrointestinal tract, but the most common localization is the stomach and small bowel. Extragastrointestinal localization is rare ${ }^{4}$. EGIST is most commonly found in the omentum, mesentery and retroperitoneum, although it has also been reported in the pancreas, gallbladder, retro-vaginal septum, liver, abdominal wall, perivesical tissue, pharynx and posterior mediastinum ${ }^{5,6}$. Some authors suggest they arise from the Cajal cells ${ }^{1}$, present in the myoenteric plexus of the muscularis propria of the smooth muscle in the tubular Gl tract while others propose they are mural GISTs with extramural growth that have lost connection with the gastrointestinal wall. They usually grow to a larger size than the average GIST, presenting as large masses which may cause abdominal pain. The behavior of stromal tumors differs according to location, and there seems to be a general trend for increasingly aggressive behavior as one proceeds distally along the gastrointestinal tract.

Their diagnosis is established based on the morphology and immunophenotyping. Histologically, there are three morphological types of GISTs described: spindle cell (70\%), epithelioid (20\%) and mixed ${ }^{2}$. Approximately $95 \%$ of GISTs carry an activating somatic mutation of $\mathrm{CD} 117$ which is the product of proto-onco-gene c-KIT, a tyrosine kinase transmembrane receptor located on chromosome 4 (4q11-q12). Positive immunohistochemical staining for CD117 is a defining feature of GISTs and is mandatory for its diagnosis; in the past they were classified as sarcomas. Nevertheless, there are also GISTs that have a mutation of PDGFRA instead of c-KIT. In this case report, the initial diagnosis was a sarcoma and the patient started classic chemotherapy for sarcomas. The detailed revision by our Pathological Center confirmed positivity for CD117 and the diagnosis of GIST was established. Therefore, it is important to establish a correct pathological diagnosis to avoid the misdiagnosis of these tumors. A correct diagnosis is essential for the correct treatment of the patient.

The current and widely accepted treatment for both GISTs and EGISTs is complete resection. It is important to avoid intra-operative tumor rupture, as it increases the chances of peritoneal recurrence. The National Institutes of Health $(\mathrm{NIH})$ consensus document established a classification system for risk of malignant behavior, which ranged from very low risk to high risk and was based on tumor size and mitotic count ${ }^{7}$. In general, tumors larger than $5 \mathrm{~cm}$, with more than 5 mitoses per $50 \mathrm{HPFs}$ are considered to be high risk. In these tumors, tyrosine kinase inhibitors such as Imatinib, are being used not only for those with metastatic disease but also in the adjuvant setting ${ }^{8}$. The duration of treatment as adjuvant intent is controversial. When the tumor is not completely resected, as in this case, Imatinib 400mg is used with a palliative intent and the treatment should be maintained until disease progression. In this patient, a resection without microscopic evidence of the tumor (R0) was not achieved, therefore the patient is on palliative treatment for 10 years and she has an excellent tolerance with no side effects. Our patient has an exon 11 mutation which predicts a better prognosis. The mutation of the part of the KIT exon 11 is the most commonly seen in about two thirds of patients. A mutation, KIT exon 9, is seen in about $10 \%$ of patients, while approximately another $10 \%$ to $20 \%$ of patients have no mutations $^{9}$. Patients with exon 9 mutation seem to be more resistant to therapy and a dose upgrade to $800 \mathrm{mg}$ is usually necessary. In responder patients with exon 11 mutation the response rate described in literature is about $83 \%{ }^{10}$.

\section{Conclusion}

GIST in an unusual location is presented. This case emphasizes the good response to tyrosine kinase inhibitors, even in the case of palliative therapy. A 10-year follow up has been achieved in this patient and she continues to maintain good quality of life.

\section{Bibliography}

1. Wang X, Mori I, Tang W, Utsunomiya H, Nakamura M, Nakamura Y et al. Gastrointestinal stromal tumors: are they of Cajal cell origin? Exp Mol Pathol. 2002;72:172-7.

2. Miettinen M, Lasota J. Gastrointestinal stromal tumors: definition, clinical, histological, immunohistochemical, and molecular genetic features and differential diagnosis. Virchows Arch.2001;438:1-12.

3. Casali PG, Blay JY. On behalf of the ESMO/CONTICANET/EUROBONET Consensus Panel of Experts. Gastrointestinal stromal tumours: ESMO Clinical practice guidelines for diagnosis, treatment and follow-up. Ann Oncol. 2010;21(Suppl 5):98-102.

4. Corless CL, Fletcher JA, Heinrich MC. Biology of gastrointestinal stromal tumors. J Clin Oncol.2005;22(18):3813-25.

5. Pidhorecky I., Cheney R.T., Kraybill W.G., Gibbs J.F. Gastrointestinal stromal tumors: current diagnosis, biologic behavior, and management. Annals of Surgical Oncology. 2000;7(October (9)):705-712.

6. Castillo-Sang M., Mancho S., Tsang A.W., Gociman B., Almaroof B., Ahmed M.Y. A malignant omental extra-gastrointestinal stromal tumor on a young man: a case report and review of the literature. World Journal of Surgical Oncology. 2008;6:50.

7. Fletcher CD, Berman JJ, Corless C, Gorstein F, Lasota J, Longley BJ et al. Diagnosis of gastrointestinal stromal tumors: A consensus approach, Human Pathology, May 2002, 459-465

8. George D. Demetri, Robert S. Benjamin, Charles D. Blanke, Jean-Yves Blay, Paolo Casali, Haesun Choi et al, NCCN Task Force Report: Optimal Management of Patients with Gastrointestinal Stromal Tumor (GIST)—Update of the NCCN Clinical Practice Guidelines, Journal of the National Cancer Comprehensive Network, volume 5, supplement 2.

9. Demetri GD. Targeting c-kit mutations in solid tumors: scientific rationale and novel therapeutic options. Semin Oncol. 2001;28(5 suppl 17):19-26.

10. Loiy Alkhatib, Omar Albtoush, Nesreen Bataineh, Kamal Gharaibeh, Ismail Matalka, Yasuharu Tokuda. Extragastrointestinal Stromal Tumor (EGIST) in the abdominal wall: Case report and literature review, nt J Surg Case Rep. 2011; 2(8): 253-255. 